\title{
Malliavin Calculus applied to Finance
}

\author{
Miquel Montero ${ }^{a}$ and Arturo Kohatsu-Higa ${ }^{b}$ \\ a Departament de Física Fonamental, Universitat de Barcelona, Diagonal, 647, \\ 08028-Barcelona, Spain \\ ${ }^{\mathrm{b}}$ Department of Economics, Universitat Pompeu Fabra, Ramón Trias Fargas \\ 25-27, 08005 Barcelona, Spain
}

\begin{abstract}
In this article, we give a brief informal introduction to Malliavin Calculus for newcomers. We apply these ideas to the simulation of Greeks in Finance. First to European-type options where formulas can be computed explicitly and therefore can serve as testing ground. Later we study the case of Asian options where close formulas are not available, and we also open the view for including more exotic derivatives. The Greeks are computed through Monte Carlo simulation.
\end{abstract}

\section{Introduction}

Malliavin Calculus is an area of research which for many years has been considered highly theoretical and technical from the mathematical point of view. In recent years it has become clear that there are various applications of Malliavin Calculus as far as the integration by parts formula is concerned. Among the fields where those techniques can become very useful it is worth mentioning Finance. Nevertheless it is still considered by the general audience and practitioners a field where is hard to grasp the basic ideas or to obtain new contributions.

In this article $^{1}$ we present an informal introduction to Malliavin Calculus which we hope can open the area to the broad scientific community, and even to practitioners. For serious mathematical approaches to the topic we refer the readers to the authoritative books on the matter, e.g. [1-3]. We have tried to take the spirit of the issues to try to explain in simple terms the elements of the theory.

$\overline{1}$ Some of the ideas and results introduced here were presented by one of us (AKH) in a talk given in the 5th. Workshop on Stochastic Numerics that took place in Kyoto (Japan) in July, 2001. 


\section{Malliavin Calculus}

\subsection{Main definitions and properties}

Malliavin Calculus applies within the framework of the stochastic processes. Let then $W=\left\{W_{t}\right\}_{t \in[0, T]}$ be a standard one-dimensional Brownian motion defined on a complete probability space $(\Omega, \mathcal{F}, P)$, where we assume that $\mathcal{F}=$ $\left\{\mathcal{F}_{t}\right\}_{t \in[0, T]}$ is generated by $W$. We will now introduce the operator $D$ and its adjoint operator, also known as the Skorokhod integral, which we will denote by $D^{*}$. Let consider a random variable of the form $F=f\left(W_{t_{1}}, \ldots, W_{t_{n}}\right)$, where $f$ is smooth enough on its arguments ${ }^{2}$. The outcome we obtain when we apply $D$ on $F$ is simply

$$
D_{t} F=\left.\sum_{i=1}^{n} \frac{\partial}{\partial x_{i}} f\left(W_{t_{1}}, \ldots, x_{i}, \ldots, W_{t_{n}}\right)\right|_{x_{i}=W_{t_{i}}} \Theta\left(t_{i}-t\right)
$$

where $\Theta(t)$ is the Heaviside step function. For stochastic processes $u=\left\{u_{t}\right\}_{t \in[0, T]}$ on $(\Omega, \mathcal{F}, P)$, the rule behaves in a similar way, just ignoring any possible explicit time dependence of $u$. Since $D$ operates on functions in the form of partial derivatives, it shares their general properties: e.g. linearity, the chain rule or the product rule.

The adjoint operator behaves as well like a stochastic integral. In fact, if $u_{t}$ is $\mathcal{F}_{t}$ adapted, that is, if $u_{t}$ depends only on $W=\left\{W_{s}\right\}_{s \in[0, t]}$, then $D^{*}(u)=$ $\int_{0}^{T} u_{t} d W_{t}$, is just the Itô integral of $u$; see e.g. [2]. Here we write $D^{*}(u)=$ $\int_{0}^{T} u_{t} d W_{t}$, even if $u_{t}$ is not $\mathcal{F}_{t}$ adapted.

Both operators $D$ and $D^{*}$ are linked by the following duality principle:

$$
E\left[\int_{0}^{T}\left(D_{t} F\right) u_{t} d t\right]=E\left[F D^{*}(u)\right]
$$

An heuristic derivation of this equality can be found in Appendix A. It is not intended there to provide with a rigorous demonstration from the viewpoint of mathematics, but with a more friendly picture of what lies beneath. Formula (1) is the cornerstone on which the integration by parts formula is grounded. It will allow us to exchange the stochastic derivative acting on $F$, by a stochastic integral that does not affect it. We will show in a while how to take advantage of this expression, and how we can reduce stochastic derivatives to the most common partial derivatives.

$\overline{2}$ We will hereafter assume that all involved functions and processes are well behaved, in the sense that they fulfil those properties that the definitions and computations demand. 
Prior to that, we will introduce another formula which is worth mentioning, since it will allow us to extract random variables out of an stochastic integral:

$$
\int_{0}^{T} F u_{t} d W_{t}=F \int_{0}^{T} u_{t} d W_{t}-\int_{0}^{T}\left(D_{t} F\right) u_{t} d t .
$$

This property follows directly from equation (1) and the product rule of the derivative operator $D$. Let us assume that $F$ an $G$ are any two stochastic variables, and $u_{t}$ a generic process, then

$$
\begin{aligned}
E\left[G F D^{*}(u)\right] & =E\left[\int_{0}^{T}\left(D_{t} G F\right) u_{t} d t\right] \\
& =E\left[\int_{0}^{T} G\left(D_{t} F\right) u_{t} d t\right]+E\left[\int_{0}^{T}\left(D_{t} G\right) F u_{t} d t\right] \\
& =E\left[G \int_{0}^{T}\left(D_{t} F\right) u_{t} d t\right]+E\left[G D^{*}(F u)\right]
\end{aligned}
$$

which implies that

$$
E\left[G D^{*}(F u)\right]=E\left[G\left(F D^{*}(u)-\int_{0}^{T}\left(D_{t} F\right) u_{t} d t\right)\right],
$$

for any random variable $G$. Therefore equation (2) must hold.

\subsection{Useful examples}

A gentler introduction may say that the idea behind the operator $D$ is to differentiate a random variable with respect to the underlying noise being this generated by the Wiener process $W$. Therefore heuristically one may think that $D_{s} "=" \frac{\partial}{\partial\left(d W_{s}\right)}$. With this in mind one can guess how to differentiate various random variables without concerning the more formal definition that we have previously quoted. Thus, some examples could be:

$$
\begin{aligned}
D_{s} W_{t} & =\Theta(t-s), \\
D_{s} f\left(W_{t}\right) & =f^{\prime}\left(W_{t}\right) \Theta(t-s), \text { and } \\
D_{s}\left(\int_{0}^{T} f\left(W_{u}\right) d W_{u}\right) & =\int_{s}^{T} f^{\prime}\left(W_{u}\right) d W_{u}+f\left(W_{s}\right),
\end{aligned}
$$

among others.

A way to understand how Malliavin Calculus can be helpful to financial problems is introducing the following integration by parts procedure, designed on the basis of the duality principle in (1). Let us assume that we are trying to 
compute $E\left[f^{\prime}(X) Y\right]$, where $X$ and $Y$ are two random variables. Suppose now that we are not able to solve this problem directly, for instance, because the join probability density function of $X$ and $Y$ is unknown. Moreover, the possibility of obtaining the answer through simulation is not a suitable or even feasible way, due to the presence of the derivative on function $f$. In this scenario we could use the formalism we have presented so far, in order to remove the derivative from $f$ :

$$
E\left[f^{\prime}(X) Y\right]=E[f(X) H]
$$

in exchange for the appearing of some new random variable $H$.

Therefore, let us begin just applying the $D$ operator on $Z, Z=f(X)$. The chain rule dictates that

$$
D_{s} Z=f^{\prime}(X) D_{s} X
$$

Now we need to add $Y$ to this expression. Thus, we multiply the above by $Y h(s)$ where $h$ is an arbitrary process - maybe depending on $X, Y$ or even on another random variable - to be chosen appropriately. Then

$$
Y h(s) D_{s} Z=f^{\prime}(X) Y h(s) D_{s} X .
$$

Integrating this for $s \in[0, T]$, we have that

$$
\begin{gathered}
\int_{0}^{T} Y h(s) D_{s} Z d s=\int_{0}^{T} f^{\prime}(X) Y h(s) D_{s} X d s=f^{\prime}(X) Y \int_{0}^{T} h(s) D_{s} X d s, \text { then } \\
f^{\prime}(X) Y=\int_{0}^{T} \frac{Y h(s) D_{s} Z}{\int_{0}^{T} h(v) D_{v} X d v} d s, \text { and therefore } \\
E\left[f^{\prime}(X) Y\right]=E\left[\int_{0}^{T}\left(D_{s} Z\right) u_{s} d s\right]
\end{gathered}
$$

with

$$
u_{s}=\frac{Y h(s)}{\int_{0}^{T} h(v) D_{v} X d v} .
$$

Finally, we could apply equation (1) on the last expression,

$$
E\left[f^{\prime}(X) Y\right]=E\left[f(X) D^{*}\left(\frac{Y h(\cdot)}{\int_{0}^{T} h(v) D_{v} X d v}\right)\right],
$$

and thus we achieve the formula we were looking for, with $H$ equal to

$$
H \equiv D^{*}\left(\frac{Y h(\cdot)}{\int_{0}^{T} h(v) D_{v} X d v}\right) .
$$

When $h(u)$ depends at most on $X, Y$ or both of them, $H=H(X, Y)$. In particular for $h$ equal to a constant we have that

$$
H \equiv H(X, Y)=D^{*}\left(\frac{Y}{\frac{1}{T} \int_{0}^{T} D_{v} X d v}\right) .
$$


If one has higher order derivatives then one has to repeat this procedure iteratively. We must however ensure that the above expectations are all finite (in particular the ones related to $H$ ). Note that the integral $\int_{0}^{T} h(v) D_{v} X d v$ should not be degenerate with probability one. Otherwise the above argument is bound to fail. The process $h$ that appears in this calculation is a parameter process that can be chosen so as to obtain this non-degeneracy. In the particular case that $h(v) \propto D_{v} X$ one obtains the so called Malliavin covariance matrix.

In conclusion one can build different integration by parts formulas depending on how we choose this process $h$. In the next section we use this formula in order to apply it to a concrete problem in Finance.

\section{Greeks in Finance}

European options are contracts that are signed between two parties (usually a bank and a customer) that allows to obtain certain monetary benefits if the price of certain asset fall above (call option) or below (put option) a certain fixed value, the strike price, at a certain fixed date, the expiration time. A Greek is the derivative of an option price with respect to a parameter. In general, let $X \equiv X(\alpha)$ be a random variable that depends on a parameter $\alpha$. Suppose that the option price is computed through a payoff function in the following form $\mathcal{P}(\alpha)=E[\Phi(X(\alpha), \alpha)]$ where $\Phi$ is generally non-smooth. A Greek is therefore a measure of the sensibility of this price with respect to its parameters. In particular, it could serve to prevent future dangers in the position of a company holding these options. The problem of computing Greeks in Finance has been studied by various authors: [4-8], among others. Let us take a closer look at the problem. If the Leibnitz rule of interchange between expectation and differentiation were true then we would have

$$
\frac{\partial \mathcal{P}(\alpha)}{\partial \alpha}=\frac{\partial E[\Phi(X(\alpha), \alpha)]}{\partial \alpha}=E\left[\Phi^{\prime}(X(\alpha), \alpha) \frac{\partial X(\alpha)}{\partial \alpha}+\frac{\partial \Phi(X(\alpha), \alpha)}{\partial \alpha}\right]
$$

When the above expression does not have a close formula then one may start thinking in performing some Monte Carlo simulations in order to approximate the above quantity. If $\Phi$ is somewhat regular then we can use the last expression above to do this: we shall call this procedure the "direct method". Unfortunately in various cases $\Phi$ is not differentiable. Then one can resort to the use of the middle expression above to generate what is known as finite difference method. This method has been somewhat successful in the recent past but we would like to discuss here the application of the integration by parts presented before in order to compute these derivatives. 


\section{The European-style options}

We shall illustrate how this procedure works by choosing a very special subset of the large family of Financial derivatives: what we have called European-style options. In this class of derivatives we will find all the options whose payoff function depends only on the value of the underlying at the expiration time $T$, which is previously fixed. Examples of the European-type options are the vanillas - the more classical European calls and puts - , or the binaries - the so called "cash-or-nothing" options - , among others. These options will differ, for instance, from the American-style options, where the execution time is not fixed but belongs to an interval; and also from the Asian-style options where the payoff depends on some average of the value of the asset in a given period of time. We will return on this topic afterwards.

The interest of the European-style options is that they are a class of derivatives whose Greeks can be computed in closed form for particular classes of payoff functions. The reason, as we will show, is that we explicitly know the probability density function of the random variable involved, $S_{T}$, whereas in other scenarios this is not true. This peculiarity provides us with a framework where we can easily test how Malliavin Calculus applies to the computation of Greeks. Later, we will also make a comment on a case where this closed formulas are not available and where this technique may prove useful.

\subsection{The Malliavin expressions}

Let us start deriving the formal expressions for some of the Greeks we shall deal with.

First we assume that our underlying asset $S$ is described by a geometric Brownian motion under the risk neutral probability $\mathbf{P}$ :

$$
S_{t}=S_{0}+\int_{0}^{t} r S_{s} d s+\int_{0}^{t} \sigma S_{s} d W_{s}
$$

where $r$ is the interest rate and $\sigma$ is the volatility. This model is one of the models typically used to describe stock prices or stock indices.

Second, from the previous arguments it follows that $X(\alpha)$ must be in general a functional of $S$. In the case of European-type options, $X(\alpha)=S_{T}$ and from (8):

$$
S_{T}=S_{0} e^{\left\{\mu T+\sigma W_{T}\right\}},
$$

where $\left\{W_{t}\right\}_{t \in[0, T]}$ is the Wiener process, and $\mu$ is just $r-\sigma^{2} / 2$. Expression (9) is involved in all the following derivations. 
Now we can compute Delta, $\Delta$, the first partial derivative of the (discounted) expected outcome of the option, with respect to the present value of the asset:

$$
\Delta=\frac{\partial}{\partial S_{0}} E\left[e^{-r T} \Phi\left(S_{T}\right)\right]=\frac{e^{-r T}}{S_{0}} E\left[\frac{\partial S_{T}}{\partial S_{0}} \Phi^{\prime}\left(S_{T}\right)\right]=\frac{e^{-r T}}{S_{0}} E\left[\Phi^{\prime}\left(S_{T}\right) S_{T}\right] .
$$

Now we may perform the integration by parts applying the formula given in $(6)$,

$$
\Delta=\frac{e^{-r T}}{S_{0}} E\left[\Phi\left(S_{T}\right) D^{*}\left(\frac{S_{T}}{\int_{0}^{T} D_{v} S_{T} d v}\right)\right]
$$

which removes the derivative of $\Phi$ from the expectation.

The integral term in the denominator of the last expression will appear many times along our exposition. In order to compute it we must remember the rules of the stochastic derivative stated above:

$$
D_{u} S_{T}=\sigma S_{T} D_{u} W_{T}=\sigma S_{T} \Theta(T-u),
$$

and then

$$
\int_{0}^{T} D_{u} S_{T} d u=\sigma T S_{T}
$$

Then we are able to perform the stochastic integral in (10),

$$
D^{*}\left(\frac{S_{T}}{\int_{0}^{T} D_{v} S_{T} d v}\right)=D^{*}\left(\frac{S_{T}}{\int_{0}^{T} \sigma S_{T} d v}\right)=D^{*}\left(\frac{1}{\sigma T}\right)=\frac{W_{T}}{\sigma T},
$$

with the help of equation (2) applied to $F=\frac{1}{\sigma T}$. Then the expression for $\Delta$ reads,

$$
\Delta=e^{-r T} E\left[\Phi\left(S_{T}\right) \frac{W_{T}}{S_{0} \sigma T}\right]
$$

Of course, we could have started from equation (4) instead of (6). In such a case the expression for $\Delta$ can be reduced to

$$
\Delta=\frac{e^{-r T}}{S_{0} \sigma} E\left[\Phi\left(S_{T}\right) D^{*}\left(\frac{h(\cdot)}{\int_{0}^{T} h(v) d v}\right)\right]
$$

just applying the recipe that appears in (11). In order to progress further we must specify $h(u)$ a little more. Thus, if $h(u)$ is, for instance, a deterministic function, we will have

$$
\Delta=\frac{e^{-r T}}{S_{0} \sigma} E\left[\Phi\left(S_{T}\right) \frac{\int_{0}^{T} h(u) d W_{u}}{\int_{0}^{T} h(v) d v}\right]
$$

whereas the picture varies when $h(u)$ is a stochastic process. In particular, if we set $h(u)=S_{u}$, the final equation for the Greek reads

$$
\Delta=\frac{e^{-r T}}{S_{0}} E\left[\Phi\left(S_{T}\right)\left(\frac{\int_{0}^{T} S_{u} d W_{u}}{\sigma \int_{0}^{T} S_{v} d v}+\frac{1}{2}\right)\right]=\frac{e^{-r T}}{S_{0} \sigma^{2}} E\left[\Phi\left(S_{T}\right)\left(\frac{S_{T}-S_{0}}{\int_{0}^{T} S_{v} d v}-\mu\right)\right]
$$


We must point out that all the expressions under the expectation signs are unbiased estimates of $\Delta$. Under this light no one is better than the others. We can classify them however after computing their variances. From this point of view, the best estimate is, of course, the one with lower variance - see the discussion in Appendix B as well. Let us illustrate this just exploring the choice of function $h(u)$ in equation (13). First of all, we will introduce $g(u)=h(u) / \int_{0}^{T} h(v) d v$, as the key magnitude to tune, since

$$
\Delta=\frac{e^{-r T}}{S_{0} \sigma} E\left[\Phi\left(S_{T}\right) \int_{0}^{T} g(u) d W_{u}\right] .
$$

The variance of the estimate depends only on $g(u)$ through

$$
E\left[\left(\frac{e^{-r T}}{S_{0} \sigma} \Phi\left(S_{T}\right) \int_{0}^{T} g(u) d W_{u}\right)^{2}\right]
$$

because it shows no bias. The minimum of this expression will be achieve when $E\left[\left(\int_{0}^{T} g(u) d W_{u}\right)^{2} \mid S_{T}\right]$ is as small as possible. In the present framework $g(u)$ is just a deterministic function that can cross the conditional expectation sign, leaving only a computation that involves the differentials of the Wiener process. But even in this case, when $W_{T}$ is settled, the increments are delta correlated, i.e. $E\left[d W_{u} \cdot d W_{v} \mid S_{T}\right]=\delta(u-v) d u d v$. Thus, we want to minimize $\int_{0}^{T} g^{2}(u) d u$, under the following restriction: $\int_{0}^{T} g(u) d u=1$. The Lagrange multipliers technique, in which one search extreme values of $\mathcal{L}$ with no constrains,

$$
\mathcal{L}=\int_{0}^{T} g^{2}(u) d u-\lambda\left(\int_{0}^{T} g(u) d u-1\right),
$$

is the most convenient choice in order to solve this kind of problems. In the present case the right answer turns out to be $g(u)=1 / T$. We will use this convention for $g(u)$, and thus for $h(u)$, unless we explicitly state another prescription.

Let us move now into a new Greek: Vega. It measures how sensitive is the price of the option when the volatility changes,

$$
\mathcal{V}=\frac{\partial}{\partial \sigma} E\left[e^{-r T} \Phi\left(S_{T}\right)\right]=e^{-r T} E\left[\frac{\partial S_{T}}{\partial \sigma} \Phi^{\prime}\left(S_{T}\right)\right]=e^{-r T} E\left[S_{T}\left(W_{T}-\sigma T\right) \Phi^{\prime}\left(S_{T}\right)\right] .
$$

We invoke again the recipe in Section 2 and thus we can withdraw the derivative form $\Phi\left(S_{T}\right)$,

$$
\mathcal{V}=e^{-r T} E\left[\Phi\left(S_{T}\right) D^{*}\left(\frac{S_{T}\left(W_{T}-\sigma T\right)}{\int_{0}^{T} D_{v} S_{T} d v}\right)\right]=e^{-r T} E\left[\Phi\left(S_{T}\right) D^{*}\left(\frac{W_{T}}{\sigma T}-1\right)\right],
$$


where we have used the expression (11). So the computation we must face is

$$
D^{*}\left(\frac{W_{T}}{\sigma T}-1\right)=\frac{1}{\sigma T} D^{*}\left(W_{T}\right)-W_{T}
$$

Here a new instance of stochastic integral appears, $D^{*}\left(W_{T}\right)$. The rule which we must take into account in order to solve the problem is again in (2), with $F=W_{T}$,

$$
D^{*}\left(W_{T}\right)=W_{T}^{2}-\int_{0}^{T} D_{s} W_{T} d s=W_{T}^{2}-T,
$$

what lead us finally to this expression for $\mathcal{V}$,

$$
\mathcal{V}=E\left[e^{-r T}\left\{\frac{W_{T}^{2}}{\sigma T}-W_{T}-\frac{1}{\sigma}\right\} \Phi\left(S_{T}\right)\right] .
$$

The last example we will present here is one involving a second derivative: Gamma. $\Gamma$ inform us on the second order dependence of the price of the option on the actual value of the underlying,

$$
\Gamma=\frac{\partial^{2}}{\partial S_{0}^{2}} E\left[e^{-r T} \Phi\left(S_{T}\right)\right]=\frac{e^{-r T}}{S_{0}^{2}} E\left[S_{T}^{2} \Phi^{\prime \prime}\left(S_{T}\right)\right] .
$$

After a first integration by parts we obtain,

$$
\Gamma=\frac{e^{-r T}}{S_{0}^{2}} E\left[\Phi^{\prime}\left(S_{T}\right) D^{*}\left(\frac{S_{T}^{2}}{\int_{0}^{T} D_{v} S_{T} d v}\right)\right]=\frac{e^{-r T}}{S_{0}^{2}} E\left[\Phi^{\prime}\left(S_{T}\right) D^{*}\left(\frac{S_{T}}{\sigma T}\right)\right] .
$$

The stochastic integral may be simplified using once more formula (2) on $F=\frac{S_{T}}{\sigma T}$, leading to

$$
D^{*}\left(\frac{S_{T}}{\sigma T}\right)=\frac{S_{T}}{\sigma T} D^{*}(1)-\frac{1}{\sigma T} \int_{0}^{T} D_{s} S_{T} d s=S_{T}\left\{\frac{W_{T}}{\sigma T}-1\right\} .
$$

Afterwards we can perform the second integration by parts, yielding:

$\Gamma=\frac{e^{-r T}}{S_{0}^{2}} E\left[\Phi^{\prime}\left(S_{T}\right) S_{T}\left\{\frac{W_{T}}{\sigma T}-1\right\}\right]=\frac{e^{-r T}}{S_{0}^{2}} E\left[\Phi\left(S_{T}\right) D^{*}\left(\frac{S_{T}}{\int_{0}^{T} D_{v} S_{T} d v}\left\{\frac{W_{T}}{\sigma T}-1\right\}\right)\right]$.

The stochastic integral is now slightly cumbersome, but it does not endow any complexity that we have not seen before,

$$
D^{*}\left(\frac{S_{T}}{\int_{0}^{T} D_{v} S_{T} d v}\left\{\frac{W_{T}}{\sigma T}-1\right\}\right)=\frac{1}{\sigma T} D^{*}\left(\frac{W_{T}}{\sigma T}-1\right)=\frac{1}{\sigma T}\left\{\frac{W_{T}}{\sigma T}-W_{T}-\frac{1}{\sigma}\right\} .
$$

If we bring together the previous partial results we will obtain the expression,

$$
\Gamma=E\left[\frac{e^{-r T}}{S_{0}^{2} \sigma T}\left\{\frac{W_{T}}{\sigma T}-W_{T}-\frac{1}{\sigma}\right\} \Phi\left(S_{T}\right)\right] .
$$


If we compare it with (14), we find the following relationship between $\mathcal{V}$ and $\Gamma$ :

$$
\Gamma=\frac{\mathcal{V}}{S_{0}^{2} \sigma T}
$$

Since we have indeed closed expressions for all the Greeks, we may easily check the correctness of the above statements. We shall recover property (16) of the European-style options in the next section. The above identities are very well known by practitioners although their proofs do not usually recall the integration by parts formula in the form we have introduced it here.

\subsection{The explicit computation}

The reason for the existence of such expressions for the Greeks of Europeantype options is due to the fact that there is a closed and tractable formula for the probability density function of $S_{T}$. This is the lognormal distribution that is written as

$$
p(x)=\frac{1}{x \sqrt{2 \pi \sigma^{2} T}} \exp \left\{-\left[\log \left(x / S_{0}\right)-\mu T\right]^{2} / 2 \sigma^{2} T\right\} .
$$

When $p(x)$ is available we can face the problem from a different perspective. In this case we are able to compute all the partial derivatives, starting from the explicit formulation for the price of the option, $\mathcal{P}$,

$$
\mathcal{P}=E\left[e^{-r T} \Phi\left(S_{T}\right)\right]=\int_{0}^{\infty} e^{-r T} \Phi(x) p(x) d x
$$

usually just a formal expression, which now becomes handy. We can show this computing the value of $\Delta$, in terms of partial derivatives of $p(x)$ :

$$
\Delta=\int_{0}^{\infty} e^{-r T} \Phi(x) \frac{\partial p(x)}{\partial S_{0}} d x=\int_{0}^{\infty} e^{-r T} \Phi(x) \frac{\partial \log p(x)}{\partial S_{0}} p(x) d x
$$

Note that we get an expression that can be easily rewritten in a way that apparently resembles our previous results, since one integration by parts has been implicitly done, and a kernel naturally appears,

$$
\Delta=E\left[e^{-r T} \Phi\left(S_{T}\right)\left(\frac{\partial \log p(x)}{\partial S_{0}}\right)_{x=S_{T}}\right]
$$

But we have not yet exploited the information we have about the functional form of $p(x)$,

$$
\left(\frac{\partial \log p(x)}{\partial S_{0}}\right)_{x=S_{T}}=\frac{1}{S_{0} \sigma^{2} T}\left[\log \left(x / S_{0}\right)-\mu T\right]_{x=S_{T}}=\frac{W_{T}}{S_{0} \sigma T}
$$


which leads us to the same expression we have already obtain by means of Malliavin Calculus:

$$
\Delta=E\left[e^{-r T} \Phi\left(S_{T}\right) \frac{W_{T}}{S_{0} \sigma T}\right]
$$

A similar procedure applies to the other Greeks. We will obtain Vega just replacing the $S_{0}$ with a $\sigma$ in equation (18),

$$
\mathcal{V}=E\left[e^{-r T} \Phi\left(S_{T}\right)\left(\frac{\partial \log p(x)}{\partial \sigma}\right)_{x=S_{T}}\right]
$$

and, after straightforward computations, we recover equation (14). The case of Gamma leads to an expression with a very similar flavour to what we have already seen,

$$
\Gamma=E\left[e^{-r T} \Phi\left(S_{T}\right)\left\{\left(\frac{\partial \log p(x)}{\partial S_{0}}\right)^{2}+\frac{\partial^{2} \log p(x)}{\partial S_{0}^{2}}\right\}_{x=S_{T}}\right],
$$

that yields, again, the same result presented in (15). We find therefore in this frame that the property stated in (16) is fulfilled by Vega and Gamma.

We can then conclude that when we deal with European-style options, the Malliavin-related procedures presented above are equivalent to the result we attain if we directly differentiate the probability density function.

\subsection{The vanilla options}

Besides the formal comparison with the previous case, the fact of knowing $p(x)$ allows us, in principle, to completely compute all the Greeks once a payoff function has been selected. One of the most popular choice is the European, or vanilla, call whose payoff reads,

$$
\Phi(X)=(X-K)_{+}
$$

Then the price of the option is given by the well-known formula due to Black, Scholes and Merton, see [9] for instance, and it can be easily derived the following expressions for the Greeks we have presented:

$$
\begin{aligned}
& \Delta=\frac{1}{\sqrt{2 \pi}} \int_{-\infty}^{d_{1}(K)} e^{-x^{2} / 2} d x, \\
& \mathcal{V}=S_{0} \sqrt{\frac{T}{2 \pi}} e^{-\left[d_{1}(K)\right]^{2} / 2}, \text { and } \\
& \Gamma=\frac{1}{S_{0} \sqrt{2 \pi \sigma^{2} T}} e^{-\left[d_{1}(K)\right]^{2} / 2}
\end{aligned}
$$


where

$$
d_{1}(x)=\frac{1}{\sigma \sqrt{T}}\left[\log \left(S_{0} / x\right)+\left(r+\frac{1}{2} \sigma^{2}\right) T\right]
$$

as it can be found in any textbook on financial derivatives [9]. In conclusion, we are able to compute the different Greeks using the Malliavin-related formulas, and compare them with their theoretical values. We present in Fig. 1 and 2 the result of this procedure, for a given set of parameters, after Monte Carlo simulation. Only $\Delta$ and $\mathcal{V}$ are shown, since $\Gamma$ would just be a replica of the second, due to equation (16). These examples show us how the outcome of the simulation progressively attains their own theoretical value, whereas the statistical error reduces. We notice however that the use of what we have labeled as "direct method", just performing Monte Carlo simulations starting from the rhs expression in (7), would lead to an estimator with smaller variance, and therefore a better estimation. Those estimations do not appear in the Figures, in the sake of clarity. But we must remember that this technique can only be applied when the payoff is smooth enough. In our case, when payoff follows (19), Gamma cannot be computed in this way.

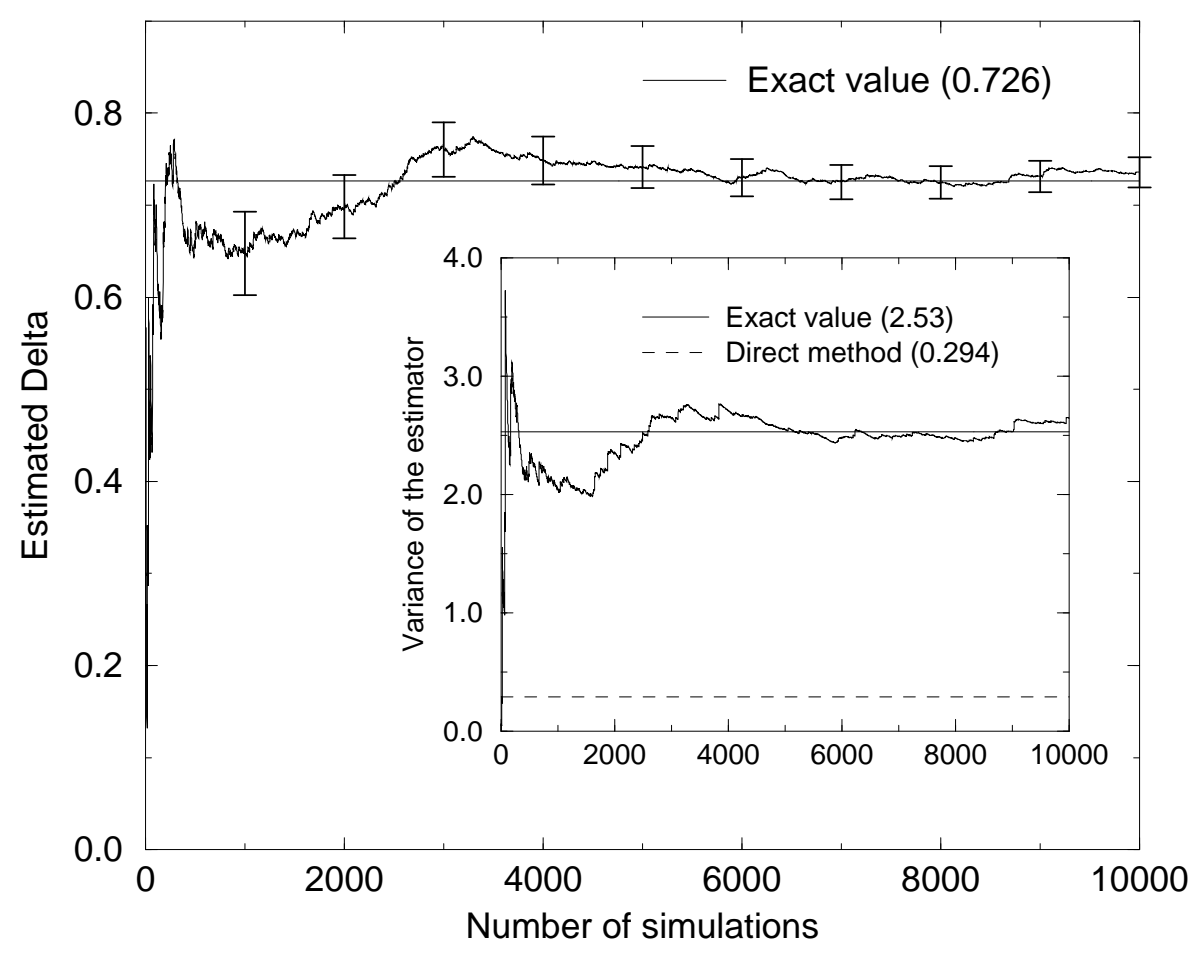

Fig. 1. Estimated value of Delta for an European call with parameters $r=0.1$, $\sigma=0.2, T=1.0$ (in years) and $S_{0}=K=100$ (in arbitrary cash units), using Monte Carlo techniques. 


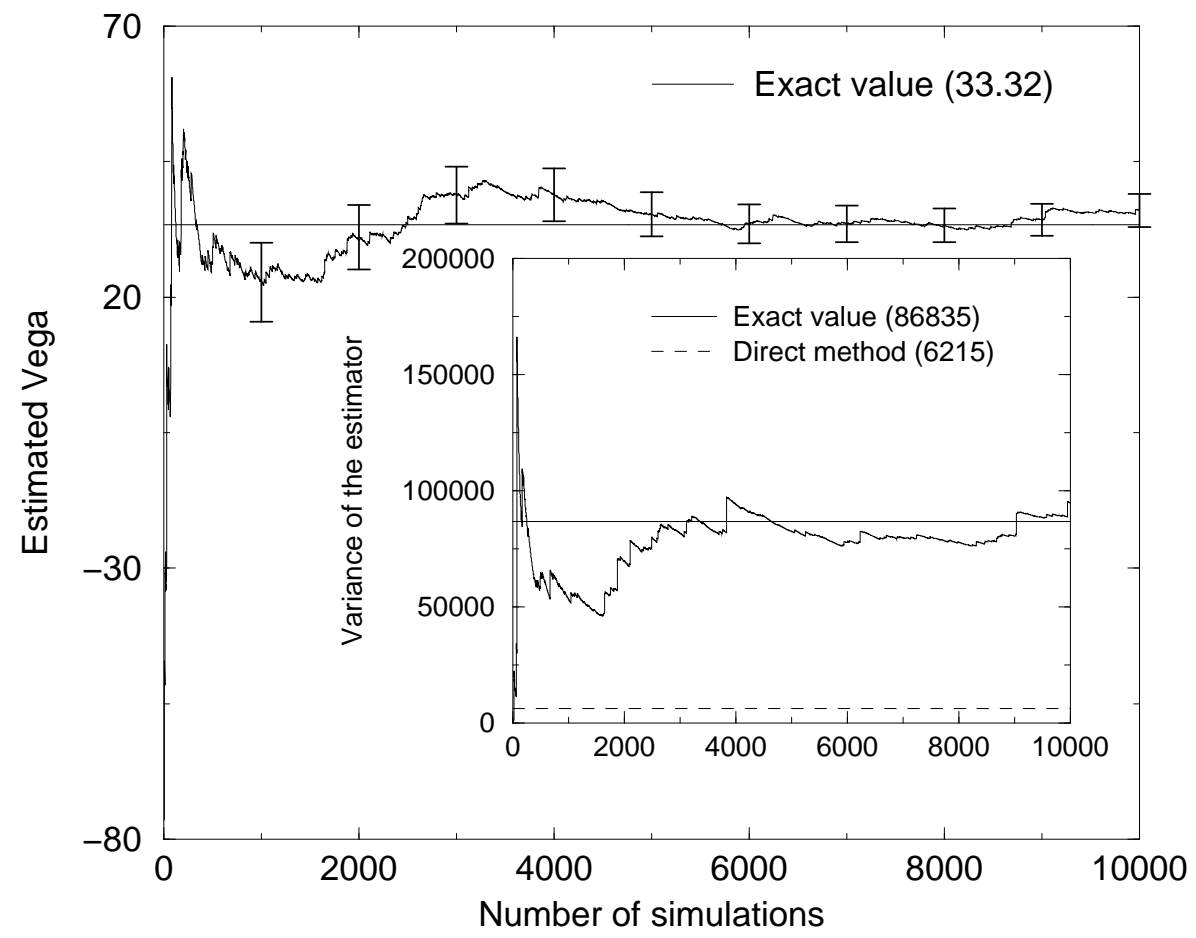

Fig. 2. Estimated value of Vega for an European call with parameters $r=0.1$, $\sigma=0.2, T=1.0$ (in years) and $S_{0}=K=100$ (in arbitrary cash units), using Monte Carlo techniques.

\section{The Asian-style options}

Here one considers the Greeks for options written on the average of the stock price $\frac{1}{T} \int_{0}^{T} S_{s} d s$, instead of the final value $S_{T}$, as in European options. Note that in this particular case the density function of the random variable does not have a known closed formula. Delta in this case is given by

$$
\Delta=\frac{\partial}{\partial S_{0}} E\left[e^{-r T} \Phi\left(\frac{1}{T} \int_{0}^{T} S_{s} d s\right)\right]=\frac{e^{-r T}}{S_{0}} E\left[\Phi^{\prime}\left(\frac{1}{T} \int_{0}^{T} S_{s} d s\right) \frac{1}{T} \int_{0}^{T} S_{u} d u\right]
$$

There are various ways of doing the integration by parts. In the already cited literature we find in [7] the following expression:

$$
\Delta=\frac{e^{-r T}}{S_{0}} E\left[\Phi\left(\frac{1}{T} \int_{0}^{T} S_{s} d s\right)\left(\frac{2 \int_{0}^{T} S_{t} d W_{t}}{\sigma \int_{0}^{T} S_{t} d t}+1\right)\right]
$$

whereas a close variant of it, which involves (8), can be found in [8]:

$$
\Delta=\frac{2 e^{-r T}}{S_{0} \sigma^{2}} E\left[\Phi\left(\frac{1}{T} \int_{0}^{T} S_{s} d s\right)\left(\frac{S_{T}-S_{0}}{\int_{0}^{T} S_{t} d t}-\mu\right)\right]
$$


Of course, we may also use the same approach we have present in the previous sections, and obtain a third one:

$$
\Delta=\frac{e^{-r T}}{S_{0}} E\left[\Phi\left(\frac{1}{T} \int_{0}^{T} S_{s} d s\right)\left(\frac{1}{<T>}\left\{\frac{W_{T}}{\sigma}+\frac{<T^{2}>}{<T>}\right\}-1\right)\right]
$$

where

$$
\begin{aligned}
<T> & =\frac{\int_{0}^{T} t S_{t} d t}{\int_{0}^{T} S_{v} d v}, \text { and } \\
<T^{2}> & =\frac{\int_{0}^{T} t^{2} S_{t} d t}{\int_{0}^{T} S_{v} d v},
\end{aligned}
$$

are something similar to a first two moments, weighted by $S(t)$. Since $S(t) \geq 0$ the similarity is more than a formal resemblance.

Although the two first expressions for $\Delta$ are statistically identical, their particular realizations when performing numerical computation will slightly differ, even though the same series of random numbers is used. The last formula is definitely a brand new estimator with its own properties, among them its smaller variance is perhaps the most relevant one. We can observe these features in Fig. 3, where we show the outcome of the Monte Carlo simulation using the three alternative instances. We have chosen again the functional form in (19) for the payoff, and the rest of parameters takes the same value we used in the making of the previous plots.

\section{Exotic derivatives}

We can apply the Malliavin formulas not only to payoff functions of a single variable, but extent the formalism in order to include also functions depending on a vector of random variables. Since we have previously introduced $S_{T}$ and $\frac{1}{T} \int_{0}^{T} S_{t} d t$, in this section we will study how to deal with a derivative whose payoff function has these two variables as arguments:

$$
\mathcal{P}=e^{-r T} E\left[\Phi\left(S_{T}, \frac{1}{T} \int_{0}^{T} S_{t} d t\right)\right] .
$$

The joint probability density function is again unknown, and therefore no closed formula will be at hand. We will try to compute the value of $\Delta$ one more time,

$$
\Delta=\frac{e^{-r T}}{S_{0}} E\left[\partial_{x} \Phi\left(S_{T}, \frac{1}{T} \int_{0}^{T} S_{t} d t\right) S_{T}+\partial_{y} \Phi\left(S_{T}, \frac{1}{T} \int_{0}^{T} S_{t} d t\right) \frac{1}{T} \int_{0}^{T} S_{t} d t\right]
$$




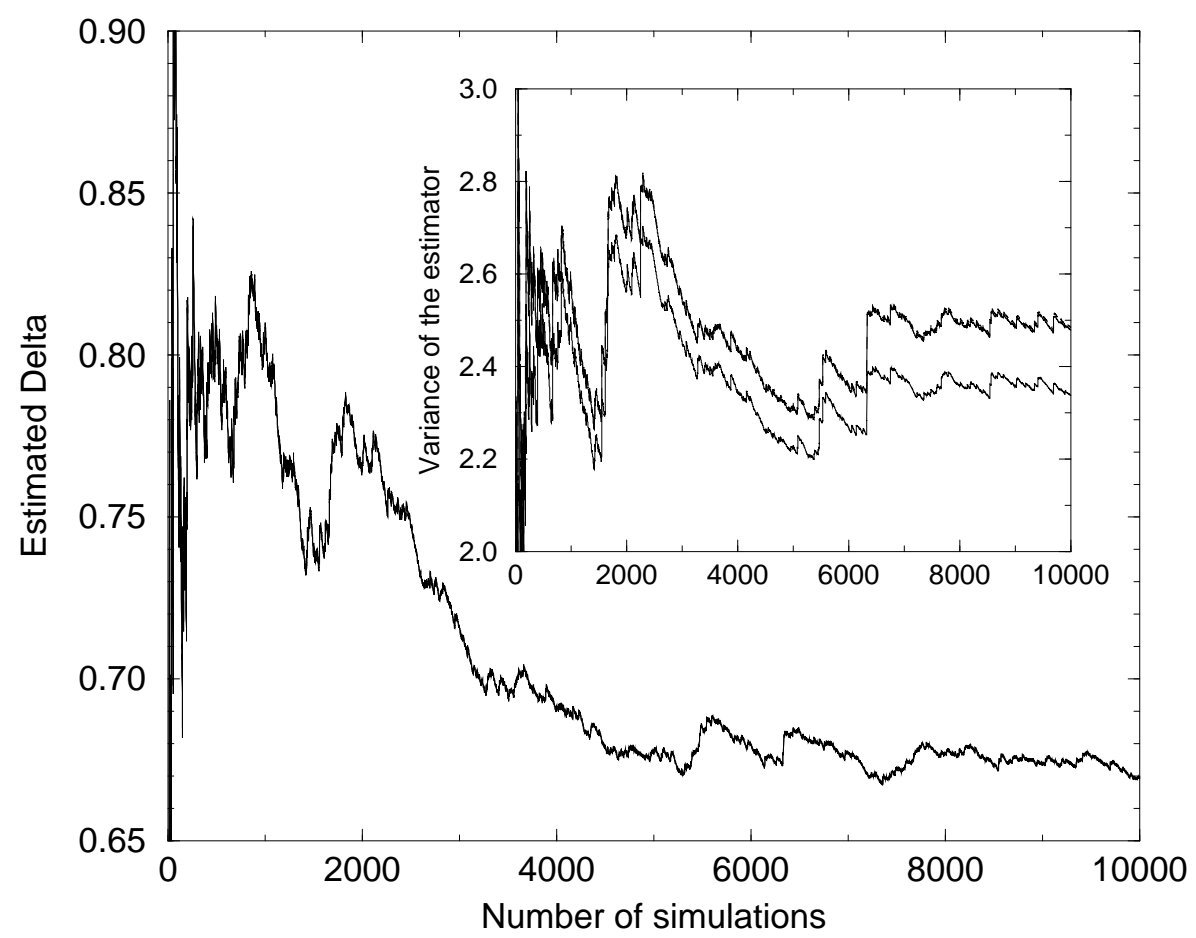

Fig. 3. Computed value of Delta for an Asian call with parameters $r=0.1, \sigma=0.2$, $T=1.0$ (in years) and $S_{0}=K=100$ (in arbitrary cash units), using Monte Carlo techniques, for the estimators presented in the main text. We have broken the interval of integration in 252 pieces, representing the approximate number of trading days in a year. The exact result turns out to be near the bottom of the graph, at about 0.65 .

where $\partial_{x} \Phi$ stands for the partial derivative of $\Phi$ with respect to their first argument, and similarly $\partial_{y} \Phi$ is the partial derivative of $\Phi$ with respect to the second one. The main difficulty of this problem is the following: when the $D$ operator is applied on $\Phi$, the terms appearing beside the partial derivatives differ from those in expression (21),

$$
D_{u} \Phi\left(S_{T}, \frac{1}{T} \int_{0}^{T} S_{t} d t\right)=\sigma\left(\partial_{x} \Phi S_{T}+\partial_{y} \Phi \frac{1}{T} \int_{u}^{T} S_{t} d t\right)
$$

We must thus manipulate the last formula in order to recover the right factors. In essence, we must find a convenient process $h(u)$ and integrate it together with $D_{u} \Phi$. For instance, we can recall that

$$
\int_{0}^{T} d u S_{u} \int_{u}^{T} S_{t} d t=\frac{1}{2}\left(\int_{0}^{T} S_{u} d u\right)^{2}
$$


and set $h(u)=S_{u}$. This operation returns the right random variables with wrong numerical factors:

$$
\frac{1}{T} \int_{0}^{T} d u S_{u} D_{u} \Phi=\sigma\left\{\partial_{x} \Phi S_{T} \frac{1}{T} \int_{0}^{T} S_{u} d u+\partial_{y} \Phi \frac{1}{2}\left(\frac{1}{T} \int_{0}^{T} S_{u} d u\right)^{2}\right\}
$$

We can however proceed in the marked direction, choosing another alike process, $h(u)=S_{u} \int_{u}^{T} S_{t} d t$, what leads to

$$
\frac{1}{T^{2}} \int_{0}^{T} d u S_{u} \int_{u}^{T} S_{t} d t D_{u} \Phi=\sigma\left\{\partial_{x} \Phi S_{T} \frac{1}{2}\left(\frac{1}{T} \int_{0}^{T} S_{u} d u\right)^{2}+\partial_{y} \Phi \frac{1}{3}\left(\frac{1}{T} \int_{0}^{T} S_{u} d u\right)^{3}\right\}
$$

where the following relationship

$$
\int_{0}^{T} d u S_{u} \int_{u}^{T} S_{t} d t \int_{u}^{T} S_{s} d s=\frac{1}{3}\left(\int_{0}^{T} S_{u} d u\right)^{3}
$$

was used. We have now two linear-independent combinations of the partial derivatives of $\Phi$, which can be added to achieve the one we were looking for

$$
\Delta=\frac{e^{-r T}}{S_{0} \sigma} E\left[\int_{0}^{T} d u\left(\frac{6 S_{u} \int_{u}^{T} S_{t} d t}{\left(\int_{0}^{T} S_{s} d s\right)^{2}}-\frac{2 S_{u}}{\int_{0}^{T} S_{s} d s}\right) D_{u} \Phi\right]
$$

Now, we can safely apply the duality principle in (1) and remove the derivative from the payoff function:

$$
\Delta=\frac{e^{-r T}}{S_{0} \sigma} E\left[\Phi D^{*}\left(\frac{6 S \cdot \int_{.}^{T} S_{t} d t}{\left(\int_{0}^{T} S_{s} d s\right)^{2}}-\frac{2 S .}{\int_{0}^{T} S_{s} d s}\right)\right]
$$

We have used on this expression the same tools we have yet shown, and we have finally obtained the next equation:

$$
\Delta=\frac{e^{-r T}}{S_{0} \sigma^{2}} E\left[\Phi\left(\frac{6 \int_{0}^{T} S_{t}^{2} d t}{\left(\int_{0}^{T} S_{s} d s\right)^{2}}-\frac{4 S_{0}+2 S_{T}}{\int_{0}^{T} S_{s} d s}-r\right)\right]
$$

Unfortunately, the presence of the term $\int_{0}^{T} S_{t}^{2} d t$ under the expectation sign marks this formula as a non-optimal expression for estimating $\Delta$ - see again the discussion in Appendix B.

Note that equation (23) will give a unbiased (although non-optimal) estimation of $\Delta$ in the case of pure European or Asian options. Let us explore the possible links between the three flavours of derivatives which we have intro- 
duced here. We set the payoff function equal to

$$
\Phi\left(S_{T}, \frac{1}{T} \int_{0}^{T} S_{t} d t\right)=\max \left(S_{T}-K, \frac{1}{T} \int_{0}^{T} S_{t} d t-K, 0\right),
$$

which reduces to equation (19) with argument $S_{T}$ when $S_{T} \geq \int_{0}^{T} S_{t} d t / T$, and with argument $\int_{0}^{T} S_{t} d t / T$ in the complementary case. In order words, the option we have introduced have a benefit that equals the best result that we will obtain with an European option and an Asian option, sharing they the same striking price $K$.

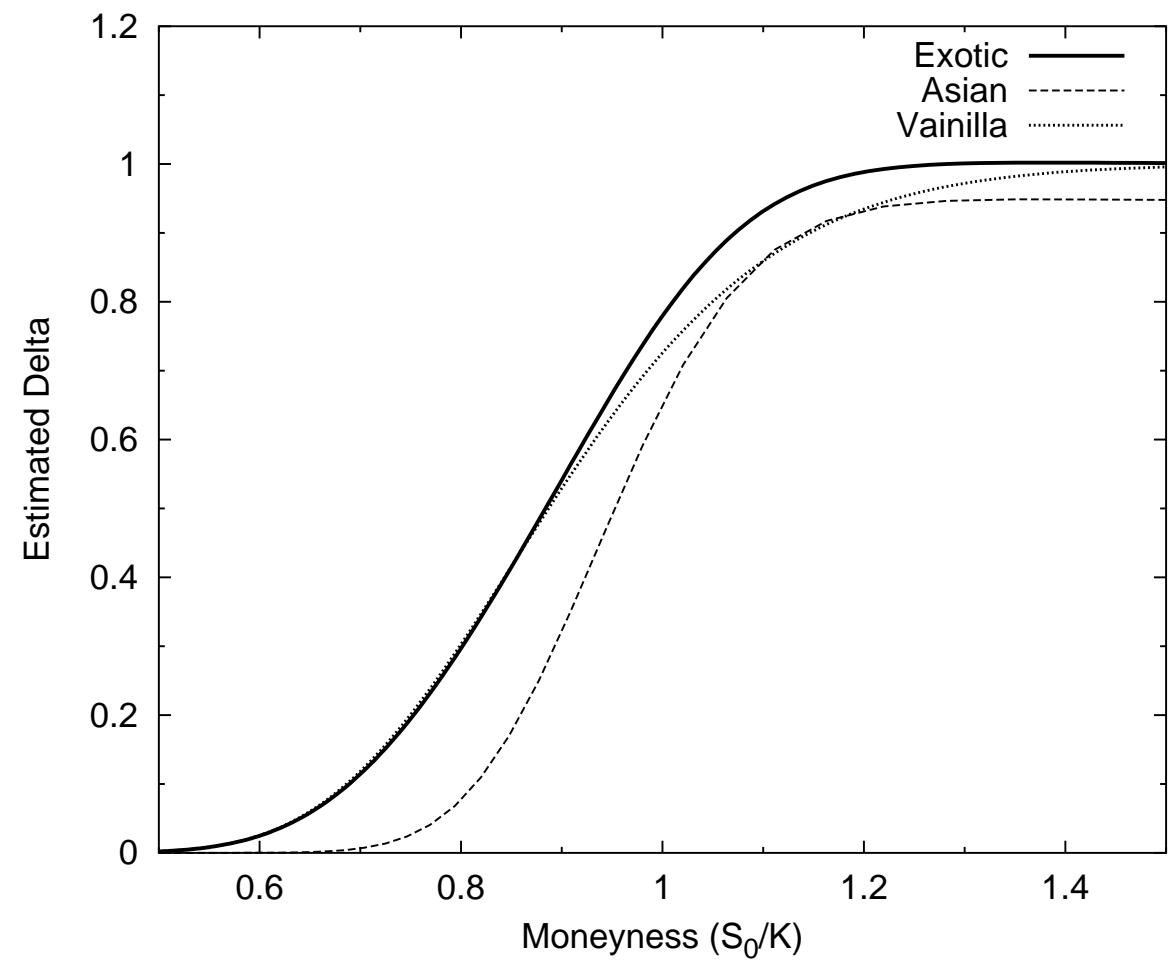

Fig. 4. Computed value of Delta for the three option flavours in terms of the present moneyness $\left(S_{0} / K\right)$. The rest of the parameter set were kept unchanged: $r=0.1$, $\sigma=0.2, T=1.0$ (in years), and the interval of integration was broken in 252 pieces again.

Obviously this new derivative should behave like an vanilla option when is more likely that the final price of the asset exceeds $K$ than the average does it. This shall be the case of options with a striking price $K$ much greater than the present price of the asset $S_{0}$, because the period at the beginning of the time interval anchors the final value of $\int_{0}^{T} S_{t} d t / T$. When we face the reverse situation, that is, when $S_{0}$ is much greater than $K$, it is a priority more feasible that the asset do not depreciate so drastically that the average falls off $K$. Summing up, we expect that the new $\Delta$ resembles the European one when $S_{0} / K$, the moneyness is below 1 , and that follows the shape of the 
Asian Delta ${ }^{3}$, if the moneyness is over 1 . In Fig. 4 we check this behaviour for the same tested set of values for the parameters.

\section{Summary}

We have presented the fundamentals of Malliavin Calculus in a friendly way, stressing their key properties. We have illustrate how these mathematical tools can be used, giving general recipes, and we have also exemplify their utility when they are applied in the field of Finance. We have tested them within the classical Black-Scholes framework, obtaining consistent results for the Greek. We have then moved into two new scenarios, analysing an Asian call and another more exotic derivative, where no closed formulas exist. In particular, we have shown how the basics of the theory we have presented here can be extended in order to solve problem involving more than just one-dimensional payoff functions.

\section{Acknowledgements}

MM has been supported in part by Dirección General de Proyectos de Investigación under contract No.BFM2000-0795, and by Generalitat de Catalunya under contract No.2000 SGR-00023.

\section{A An heuristic derivation of the duality formula}

In this appendix we will present an heuristic demonstration of equation (1). Our main purpose is to show in a intuitive way how this property works, not to present a rigorous proof from the viewpoint of Mathematics. In the first place, let us consider $W_{T}$ as a superposition of infinite-numerable Wiener processes,

$$
W_{T}=\int_{0}^{T} d W_{t}=\lim _{N \rightarrow \infty} \sum_{n=0}^{N-1} \Delta W_{n T / N}
$$

3 The value of the exotic $\Delta$ is always bigger than the European and Asian counterparts. The limit value for a plain vanilla when the moneyness tends to infinity is equal to 1 , see (20). As we have stated before, there is no closed expression for evaluating the Asian Delta. It is nevertheless easy to check that $\lim _{S_{0} / K \rightarrow \infty} \Delta=\left(1-e^{-r T}\right) / r T$. The reader can find a derivation of this result in Appendix $\mathrm{C}$. 
each of them statistically independent of the others. In fact the $\Delta W_{n T / N}$ variables, $\Delta W_{n T / N} \equiv W_{(n+1) T / N}-W_{n T / N}$, form a set of $N$ i.i.d. Gaussian variables, $\Delta W_{n T / N} \sim \mathcal{N}(0, T / N)$. In a similar way, the integral that we want to evaluate, $\int_{0}^{T}\left(D_{s} Z\right) u_{s} d s$, will be split in a infinite-numerable non-overlapping pieces:

$$
\int_{0}^{T}\left(D_{s} Z\right) u_{s} d s=\lim _{N \rightarrow \infty} \frac{T}{N} \sum_{n=0}^{N-1}\left(D_{n T / N} Z\right) u_{n T / N}
$$

The random variable $Z$ will depend on our discrete Wiener process through function $f, Z=f\left(\Delta W_{0}, \ldots, \Delta W_{(N-1) T / N}\right)$. An equivalent expression also holds for the process $u, u_{n T / N}=g\left(\Delta W_{0}, \ldots, \Delta W_{(n-1) T / N} ; n T / N\right)$. Note that we are demanding that $u$ is an adapted process. At any given time $t=n T / N$, it contains no information concerning $W_{t \in[(n+1) T / N, \ldots, T]}$, and thus it does not depends on $\Delta W_{n T / N}, \ldots, \Delta W_{(N-1) T / N}$. We will now take the expectation of expression (A.1), and we will introduce the probability density function of the Gaussian variables $\Delta W_{\frac{m T}{N}}$ :

$$
\begin{aligned}
& E\left[\int_{0}^{T}\left(D_{s} Z\right) u_{s} d s\right]= \\
& =\lim _{N \rightarrow+\infty} \prod_{m=0}^{N-1} \int_{-\infty}^{+\infty} \sqrt{\frac{N}{2 \pi T}} d\left(\Delta W_{\frac{m T}{N}}\right) e^{\left\{-\frac{N}{2 T}\left(\Delta W_{\frac{m T}{N}}\right)^{2}\right\}} \sum_{n=0}^{N-1}\left(D_{\frac{n T}{N}} Z\right) u_{\frac{n T}{N}} \frac{T}{N} \\
& =\lim _{N \rightarrow+\infty}\left(\frac{N}{T}\right)^{\frac{N}{2}-1} \sum_{n=0}^{N-1} \prod_{m \neq n} \int_{-\infty}^{+\infty} \frac{d \omega_{m}}{2 \pi} e^{-N \omega_{m}^{2} / 2 T} \int_{-\infty}^{+\infty} \frac{d \omega_{n}}{2 \pi} e^{-N \omega_{n}^{2} / 2 T} \frac{\partial Z}{\partial \omega_{n}} u_{\frac{n T}{N}}
\end{aligned}
$$

where $\omega_{m} \equiv \Delta W_{\frac{m T}{N}}$ was defined for simplicity reasons only. It is obvious that the last integral of the past expression carries all the $\omega_{n}$ dependency. Therefore we can now perform a mere integration by parts on these integral:

$$
\begin{aligned}
& \int_{-\infty}^{+\infty} d \omega_{n} e^{-N \omega_{n}^{2} / 2 T} \frac{\partial Z}{\partial \omega_{n}} u_{\frac{n T}{N}}= \\
= & \frac{N}{T} \int_{-\infty}^{+\infty} \omega_{n} Z u_{\frac{n T}{N}} e^{-N \omega_{n}^{2} / 2 T} d \omega_{n}-\int_{-\infty}^{+\infty} d \omega_{n} e^{-N \omega_{n}^{2} / 2 T} Z \frac{\partial}{\partial \omega_{n}} u_{\frac{n T}{N}} .
\end{aligned}
$$

Here we have assumed that $Z u_{\frac{n T}{N}} e^{-N \omega_{n}^{2} / 2 T} \rightarrow 0$ when $\omega_{n} \rightarrow \pm \infty$. The second term also vanishes in our case, since $\frac{\partial}{\partial \omega_{n}} u_{\frac{n T}{N}}=0$ when $u_{t}$ is adapted. If we introduce these results in (A.2) we can recover the relationship we were looking for between $D$ and its adjoint operator $D^{*}$ : 


$$
\begin{aligned}
& E\left[\int_{0}^{T}\left(D_{s} Z\right) u_{s} d s\right]= \\
& =\lim _{N \rightarrow+\infty}\left(\frac{N}{2 \pi T}\right)^{\frac{N}{2}} \prod_{m=0}^{N-1} \int_{-\infty}^{+\infty} d \omega_{m} e^{-N \omega_{m}^{2} / 2 T} Z \sum_{n=0}^{N-1} u_{\frac{n T}{N}} \omega_{n} \\
& =\lim _{N \rightarrow+\infty} E\left[Z \sum_{n=0}^{N-1} u_{\frac{n T}{N}} \Delta W_{\frac{n T}{N}}\right]=E\left[Z \int_{0}^{T} u_{s} d W_{s}\right]=E\left[Z D^{*}(u)\right] .
\end{aligned}
$$

\section{B Optimal integration}

Not all the alternative formulas we have presented throughout the present article coincide and in fact, contrary to what is claimed in [8] there is no way to obtain the integration by parts that provides the minimal variance in the case of the Asian derivatives. The main reason is that solving this problem is equivalent to finding the probability density of the random variable in question. To expose the main ideas that also appear in [8] one can note first that there is an integration by parts that is the "most" straightforward but highly unrealistic. For this, consider the generalized problem

$$
E\left[\Phi^{\prime}\left(\frac{1}{T} \int_{0}^{T} S_{s} d s\right) \frac{1}{T} \int_{0}^{T} S_{s} d s\right]=\int_{0}^{\infty} \Phi^{\prime}(x) x \psi(x) d x .
$$

Here $\psi(x)$ denotes the density of $\frac{1}{T} \int_{0}^{T} S_{s} d s$ which exists and is smooth (it is an interesting exercise of Malliavin Calculus). Therefore one can perform the integration by parts directly in the above formula thus obtaining that

$$
\begin{aligned}
& E\left[\Phi^{\prime}\left(\frac{1}{T} \int_{0}^{T} S_{s} d s\right) \frac{1}{T} \int_{0}^{T} S_{s} d s\right]=\int_{0}^{\infty} \Phi(x)\left(\psi(x)+x \psi^{\prime}(x)\right) d x \\
& E\left[\Phi\left(\frac{1}{T} \int_{0}^{T} S_{s} d s\right) \cdot\left(1+\frac{\psi^{\prime}\left(\frac{1}{T} \int_{0}^{T} S_{s} d s\right)}{\psi\left(\frac{1}{T} \int_{0}^{T} S_{s} d s\right)} \frac{1}{T} \int_{0}^{T} S_{s} d s\right)\right]
\end{aligned}
$$

Now we proceed to prove that the above gives the minimal integration by parts in the sense of variance. Obviously it is not possible to carry out the simulations unless $\psi^{\prime}$ and $\psi$ are known. Let us construct the set of all possible integration by parts. Suppose that $H$ is a random variable such that

$$
E\left[\Phi^{\prime}\left(\frac{1}{T} \int_{0}^{T} S_{s} d s\right) \frac{1}{T} \int_{0}^{T} S_{s} d s\right]=E\left[\Phi\left(\frac{1}{T} \int_{0}^{T} S_{s} d s\right) H\right]
$$


then it is not difficult to deduce that the conditional expectation of $H$ must fulfil

$$
E\left[H \mid \frac{1}{T} \int_{0}^{T} S_{s} d s\right]=1+\frac{\psi^{\prime}\left(\int_{0}^{T} S_{s} d s\right)}{\psi\left(\int_{0}^{T} S_{s} d s\right)} \frac{1}{T} \int_{0}^{T} S_{s} d s .
$$

Therefore the set of all possible integration by parts can be characterized as

$$
\mathcal{M}=\left\{H ; E\left[H \mid \frac{1}{T} \int_{0}^{T} S_{s} d s\right]=1+\frac{\psi^{\prime}\left(\frac{1}{T} \int_{0}^{T} S_{s} d s\right)}{\psi\left(\frac{1}{T} \int_{0}^{T} S_{s} d s\right)} \frac{1}{T} \int_{0}^{T} S_{s} d s\right\}
$$

Next in order we want to find the element in $Y$ that minimizes

$$
\inf _{Y \in \mathcal{M}} E\left[\Phi\left(\frac{1}{T} \int_{0}^{T} S_{s} d s\right)^{2} H^{2}\right]
$$

As in [8] is not difficult to see which $H$ achieves the minimum. This is done as follows:

$$
\begin{aligned}
& E\left[\Phi\left(\int_{0}^{T} S_{s} d s\right)^{2} H^{2}\right]= \\
& =E\left[\Phi\left(\frac{1}{T} \int_{0}^{T} S_{s} d s\right)^{2} \cdot\left(H-1-\frac{\psi^{\prime}\left(\frac{1}{T} \int_{0}^{T} S_{s} d s\right)}{\psi\left(\frac{1}{T} \int_{0}^{T} S_{s} d s\right)} \frac{1}{T} \int_{0}^{T} S_{s} d s\right)^{2}\right] \\
& +E\left[\Phi\left(\int_{0}^{T} S_{s} d s\right)^{2} \cdot\left(1+\frac{\psi^{\prime}\left(\frac{1}{T} \int_{0}^{T} S_{s} d s\right)}{\psi\left(\frac{1}{T} \int_{0}^{T} S_{s} d s\right)} \frac{1}{T} \int_{0}^{T} S_{s} d s\right)^{2}\right]
\end{aligned}
$$

due to the property of the set $\mathcal{M}$. Therefore the minimum is achieved at

$$
H=\left(1+\frac{\psi^{\prime}\left(\frac{1}{T} \int_{0}^{T} S_{s} d s\right)}{\psi\left(\frac{1}{T} \int_{0}^{T} S_{s} d s\right)} \frac{1}{T} \int_{0}^{T} S_{s} d s\right) .
$$

This is clearly impossible to write explicitly as $\psi$ is unknown in the case of Asian options. Therefore it is still an open problem to devise good ways to perform an efficient integration by parts so that the variance is made small rapidly and efficiently.

\section{Limiting value of Asian Delta}

In order to compute the value of an Asian Delta when the present moneyness tends to infinity, we will decompose the random variable in two factors:

$$
\frac{1}{T} \int_{0}^{T} S_{s} d s=S_{0} \cdot \frac{1}{T} \int_{0}^{T} Y_{s} d s
$$


The process $Y_{t}$ follows a stochastic equation equivalent to the one in (8),

$$
Y_{t}=1+\int_{0}^{t} r Y_{s} d s+\int_{0}^{t} \sigma Y_{s} d W_{s}
$$

The probability density function of $\frac{1}{T} \int_{0}^{T} Y_{s} d s, \phi(x)$, is also unknown, but contains no $S_{0}$ with certainty. Therefore the probability density function of the Asian random variable, $\psi(x)$, relates to the previous one through

$$
\psi(x)=\frac{1}{S_{0}} \phi\left(x / S_{0}\right) .
$$

The price of an Asian call with the usual payoff function $\Phi(x)=(x-K)_{+}$is,

$$
\mathcal{P}=e^{-r T} \int_{K}^{\infty}(x-K) \psi(x) d x=e^{-r T} \int_{K / S_{0}}^{\infty}\left(S_{0} y-K\right) \phi(y) d y,
$$

and, since $\Delta$ is the partial derivative of $\mathcal{P}$, with respect to $S_{0}$,

$$
\Delta=e^{-r T} \int_{K / S_{0}}^{\infty} y \phi(y) d y
$$

We can now investigate the limit of $\Delta$ when the moneyness tends to infinity:

$$
\lim _{S_{0} / K \rightarrow \infty} \Delta=e^{-r T} \int_{0}^{\infty} y \phi(y) d y=e^{-r T} E\left[\frac{1}{T} \int_{0}^{T} Y_{s} d s\right] .
$$

The expectation and the integral in the last equation can be commuted, and the computation can be followed to the very end:

$$
\lim _{S_{0} / K \rightarrow \infty} \Delta=e^{-r T} \frac{1}{T} \int_{0}^{T} d s E\left[Y_{s}\right]=\frac{1}{r T}\left(1-e^{-r T}\right)
$$

because $E\left[Y_{s}\right]=e^{r T}$.

\section{References}

[1] Ikeda N. and Watanabe S., Stochastic Differential Equations and Diffusion Processes, North-Holland, Amsterdam (1989).

[2] Nualart D., The Malliavin Calculus and Related Topics, Springer, Berlin (1995).

[3] Malliavin P., Market curvature and importance sampling of Ocone-Karatzas hedging. Conference on Stochastic Analysis, Berlin (July 2001).

[4] Broadie M. and Glasserman P., "Estimating Security Price Derivatives Using Simulation", Manag. Sci., 42, 269-285 (1996). 
[5] Benhamou E., "An application of Malliavin Calculus to continuous time Asian Options Greeks". Technical report, London School of Economics (2000).

[6] L'Ecuyer P., and Perron G., "On the convergence rates of IPA and FDC derivative estimators", Oper. Res., 42, 643-656 (1994).

[7] Fournié E., Lasry J.M., Lebuchoux J., Lions P.L., and Touzi N., "Applications of Malliavin calculus to Monte Carlo methods in finance", Finance Stochast., 3, 391-412, (1999)

[8] Fournié E., Lasry J.M., Lebuchoux J., and Lions P.L., "Applications of Malliavin calculus to Monte Carlo methods in finance. II", Finance Stochast., 5, 201-236 (2001)

[9] Wilmott, P., Derivatives, Wiley, Chichester (1998). 